\title{
Automated Test Station for the Characterization of Custom Silicon PhotoMultipliers for the Mu2e Calorimeter
}

D. Caiulo, F.Cervelli, S. Di Falco, S. Donati, L. Lucchesi, L. Morescalchi*, D. Pasciuto, E. Pedreschi, F. Raffaelli and F. Spinella

INFN - Sezione di Pisa, Pisa, Italy

E-mail: luca.morescalchi@pi.infn.it

M. Cordelli, G. Corradi, E. Diociaiuti, R. Donghia, S. Giovannella, F. Happacher, M. Martini, S, Miscetti, M. Ricci and I. Sarra

Laboratori Nazionali di Frascati dell'INFN, Frascati, Italy

\section{A. Ferrari and S. Muller}

HZDR, Helmholtz-Zentrum Dresden-Rossendorf, Germany

\section{N. Menaa and R. Sia}

Clever Operation, Orsay, France

\section{G. Pezzullo}

Yale university, New Haven, USA

\begin{abstract}
The Mu2e electromagnetic calorimeter is composed of un-doped CsI crystals coupled to large area Silicon Photomultipliers. A modular and custom SiPM layout consisting of a $3 \times 2$ array of 6x6 mm² UV-extended monolithic SiPMs has been developed to fulfill the Mu2e calorimeter requirements. After a preliminary testing phase on pre-production prototypes, Hamamatsu has been selected as vendor and the production of 4000 devices is ongoing. A detailed Quality Assurance (QA) process is being carried out on each device using an automatized test station able to measure the breakdown voltage, the gain and the dark current at three temperatures $\left(-10^{\circ} \mathrm{C}, 0^{\circ} \mathrm{C}\right.$ and $20^{\circ}$ C) and under vacuum. Up to $20 \mathrm{SiPMs}$ can be characterized at the same time. We present the design of the test station and the measurement techniques, as well as a summary of the results obtained from the first tested batches of photosensors.
\end{abstract}

Topical Workshop on Electronics for Particle Physics (TWEPP2018)

17-21 September 2018

Antwerp, Belgium

${ }^{*}$ Speaker. 


\section{Introduction}

The Mu2e electromagnetic calorimeter [1] consists of 1348 un-doped CsI crystals, each coupled to two large area Silicon Photomultipliers (SiPMs), and arranged in two disks. To reach the requested light collection while keeping a smaller total capacity, a custom SiPM package has been developed [2]: it consists of a $3 \times 2$ matrix of $6 \times 6 \mathrm{~mm}^{2}$ monolithic SiPMs (cells) with the readout organized as the parallel of 2 series of three cells. With this configuration the total capacitance becomes one third of the one of the single cell; on the other hand, the bias voltage triples. After a deep characterization of different models from different vendors [3], Hamamatsu devices (with 13360-6050CS used as cell) have been selected and a production of 4k units is ongoing. As well as ensuring the performances needed for the muon-to-electron conversion search, these photosensors have to guarantee a good reliability while operating maintenance-free in the Mu2e hostile environment [4]: any failure can only be replaced during a long technical shut-down scheduled once a year. The Quality Assurance (QA) is carried out in a dedicated soft clean room in the SiDet Fermilab department. Starting from March 2018, we are receiving one batch of 300 photosensors/month. QA is kept in phase with the production, so to check the stability of the sensors characteristics and eventually to notify to the manufacturer any reduction in the performances. As first step, each sensor is subjected to a visual inspection to detect any scratch on the resin surface or mechanical damages. Then a dimensional check is performed with a dedicated station that guarantees a 100 $\mu \mathrm{m}$ accuracy. The third step is the characterization of each sensor at the level of the single cell.

\section{Selection Criteria}

Selection criteria have been fixed to have a good uniformity between the cells of the same sensor and a light collection of at least 20 photoelectrons/MeV, as suggested by simulation [5]. Defining the operational voltage $\mathrm{V}_{\mathrm{op}}$ as $3 \mathrm{~V}$ over the breakdown voltage $\mathrm{V}_{\mathrm{br}}$, the requirements at a temperature of $20^{\circ} \mathrm{C}$ are: (i) a spread in the breakdown voltage $\mathrm{V}_{\mathrm{br}}$ between the sensor cells $<0.5 \%$; (ii) a spread in the dark current at $\mathrm{V}_{\text {op }}$ between sensor cells $<15 \%$; (iii) a gain $\times \operatorname{PDE}\left(310 \mathrm{~nm}\right.$ ) (Photon Detection Efficiency) at $\mathrm{V}_{\mathrm{op}}>0.2 \times 10^{6}$ for each cell. If a sensor does not meet these specifications it is rejected. Moreover, for each batch are also requested: (i) a Mean Time To Failure (MTTF) greater than $\sim 10^{6}$ hours/component; (ii) a maximum dark current of 2 $\mathrm{mA}$ after the exposition to a neutron fluence of $8 \times 10^{11} 1 \mathrm{MeV}(\mathrm{Si})$ eq. $\mathrm{n} / \mathrm{cm}^{2}$. These quantities are measured using a random subset of 15 samples for the MTTF and 5 samples for the neutron irradiation.

\section{Automatized Station Design}

In view of the large number of measurements to perform ( $~ 24 k$ single cell characterizations), a fully automatized station has been developed to quickly perform the task. The station consists of an automatized system able to handle fast current measurements from multiple photosensors kept at a stable temperature in the range from $-10^{\circ} \mathrm{C}$ to $20^{\circ} \mathrm{C}$. The system is controlled with a dedicated Labview software that allows the complete test 25 of sensors simultaneously without any external intervention of an operator. To avoid water vapors condensations at low temperatures, tests are 
carried out inside a vacuum vessel kept at a pressure of 100 mbar. A drawing of the station is shown in Figure 1-Left.
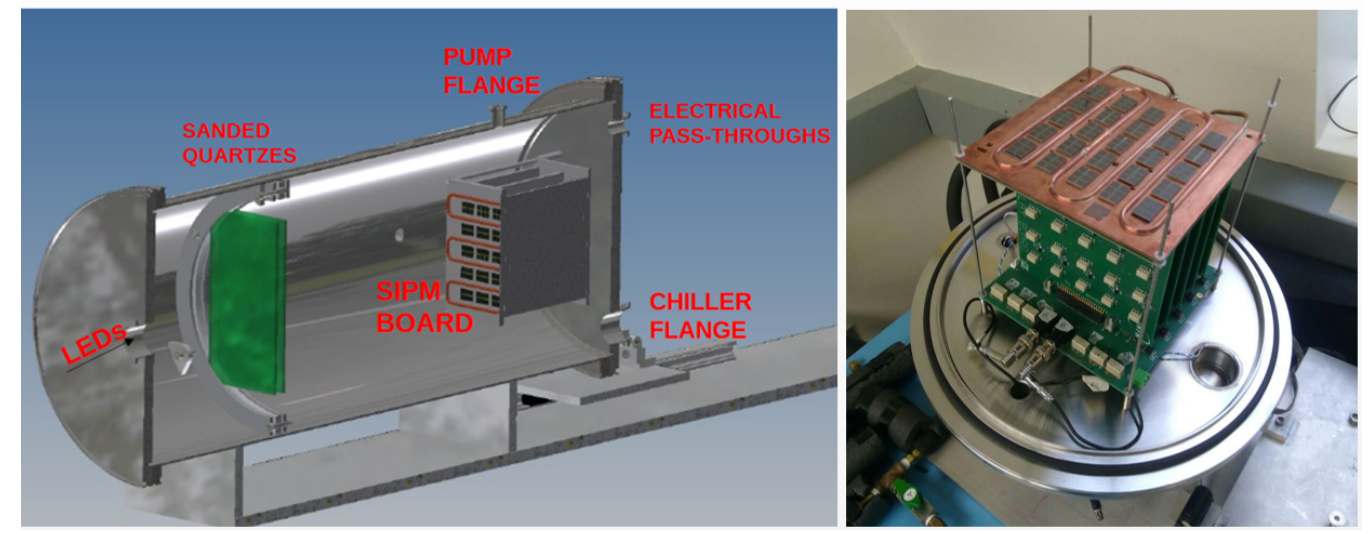

Figure 1: Left - CAD drawings of the SiPMs QA station. Right - Picture of the photosensors plugged into the cooling plate.

The photosensors under test are plugged on a copper plate (see Figure 1-Right), cooled by a chiller Julabo FL300 and posed on the top of a castle of custom PCB boards. An UV LED with the emission peaked at $310 \mathrm{~nm}$ and $\sim 1 \mathrm{~m}$ from the plate can illuminate the sensors. The light is spread by a couple of fine sanded quartzes $\sim 30 \mathrm{~cm}$ far from the LED, which is powered by a dedicated board that monitor the drained current, the voltage and the temperature. The electronics inside the vessel is composed of a PIC microcontroller that drives 110 relays to connect the wanted cell to a Keithley 6487, that provides the bias voltage and performs the current measurements. For each of the 150 cells on the plate it is performed an I-V scan to determine the breakdown voltage; thus, sensors are biased at their operational voltage and the dark current and the relative Gain $\times$ PDE are measured. This is repeated for three temperatures: $-10^{\circ} \mathrm{C}$ (backup option), $0^{\circ} \mathrm{C}$ (the operational temperature) and $20^{\circ} \mathrm{C}$ (temperature used to evaluate the technical requirements). With three points it is so possible to extrapolate the parameters in all the temperatures range. The 5 sensors posed at the corners and at the center of the board are used as reference to monitor the stability of the station, the uniformity of the light and to measure the Gain $\times$ PDE.

\section{Measurement Techniques}

The strategy that drove the station design was to reduce as much as possible the duration of a test cycle. A fast way to extract the breakdown voltage involves the analysis of the I-V curve shape. Due to the low current level around $\mathrm{V}_{\mathrm{br}}(\sim \mathrm{nA})$ in case of no illumination, the I-V scan is performed while illuminating the sensor with a low level of light so to increase the current and to obtain a more fast and precise measurement. A first estimation of the $\mathrm{V}_{\mathrm{br}}$, dependent on the incident light, is obtained by constructing the $\mathrm{d} \log (\mathrm{I}) / \mathrm{dV}$ curve and by fitting the peak position (as in [3]). This value is then used to initialize a second unbiased fit with: 


$$
I(V)= \begin{cases}\left.I_{0}+C \times\left(1-e^{-p \cdot\left(V-V_{b r}\right)}\right) \times\left(V-V_{b r}\right)\right) & V>V_{b r} \\ I_{0} & V<V_{b r}\end{cases}
$$

where $\mathrm{V}$ is the bias voltage, $\mathrm{V}_{b r}$ is the breakdown voltage, $\mathrm{I}_{0}$ is the current before the breakdown, $p$ is the triggering probability and $C$ is a factor proportional to the number of the free carriers (thermal + optical). Equation 4.1 has been obtained starting from [6] and making a couple of assumptions to limit the number of free parameters: (i) the afterpulse and the crosstalking are negligible; (ii) we are far from the second breakdown zone. To avoid regions in where the behavior of the current while the sensor is illuminated does not follow this model, the fit is performed in an interval that starts $200 \mathrm{mV}$ over $\mathrm{V}_{b r}$. An example of this procedure is shown in Figure 2, together with the difference between the quoted $\mathrm{V}_{b r}$ from the producer at $25^{\circ} \mathrm{C}$ and our technique at $20^{\circ} \mathrm{C}$.
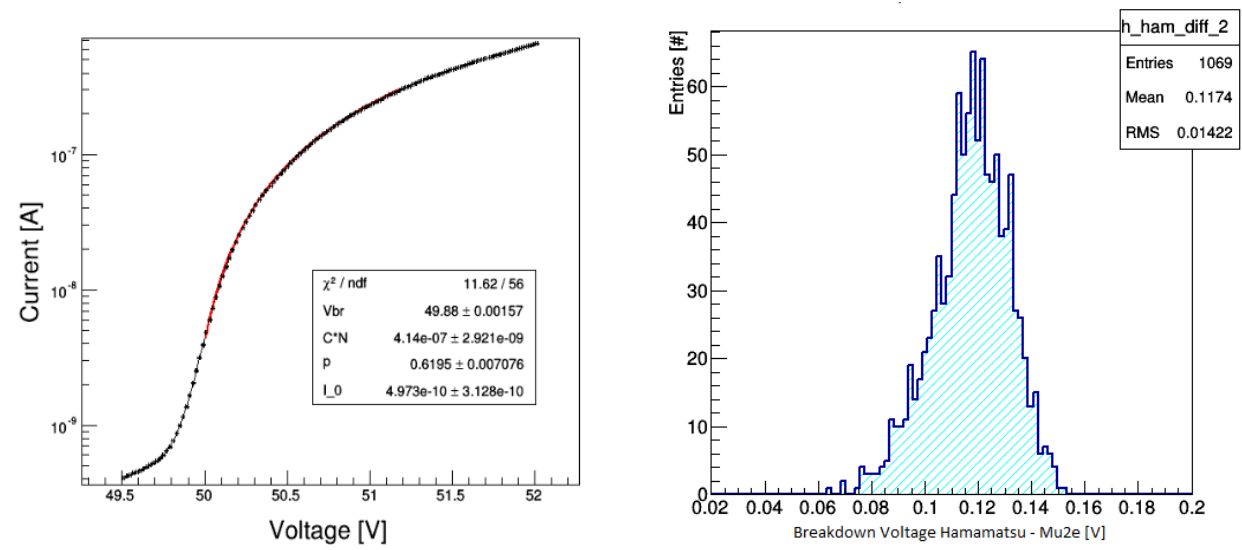

Figure 2: Left - Example of fit of the I-V curve for one cell while illuminated with a low light. Black points represents the current measurements while the red line a fit with Eq.4.1. Right - Distribution of the difference between the $\mathrm{V}_{b r}$ quoted by the producer at $25^{\circ} \mathrm{C}$ and the one obtained with our techniques at $20^{\circ} \mathrm{C}$. The nonzero mean is due partly to the different test temperatures and partly to a constant offset between the results of the two different techniques.

To measure the Gain $\times$ PDE, the LED is turned on and the five calibrated sensors in the corners and the center of the board are used as reference. The value is obtained by the ratio of the currents pulled by the sensor under test and the reference, while illuminating by the same LED light. Since the light after the diffusion is not completely uniform, an additional normalization to take into account the Gaussian light profile is applied.

The dark current is extracted by biasing the cell at the operational voltage and recording the current while the LED is turned off.

\section{First Tests Results}

The RMS distributions resulting from the characterizations of the first 4 batches are shown in Figure 3. The uniformity of the breakdown voltage inside the sensor presents a very good behavior, while the dark current has a small tail that falls out of the specifications: around the 3\% 
of the production is being rejected for this reason. All the tested sensors instead widely satisfy the requirement on the Gain $\times$ PDE.
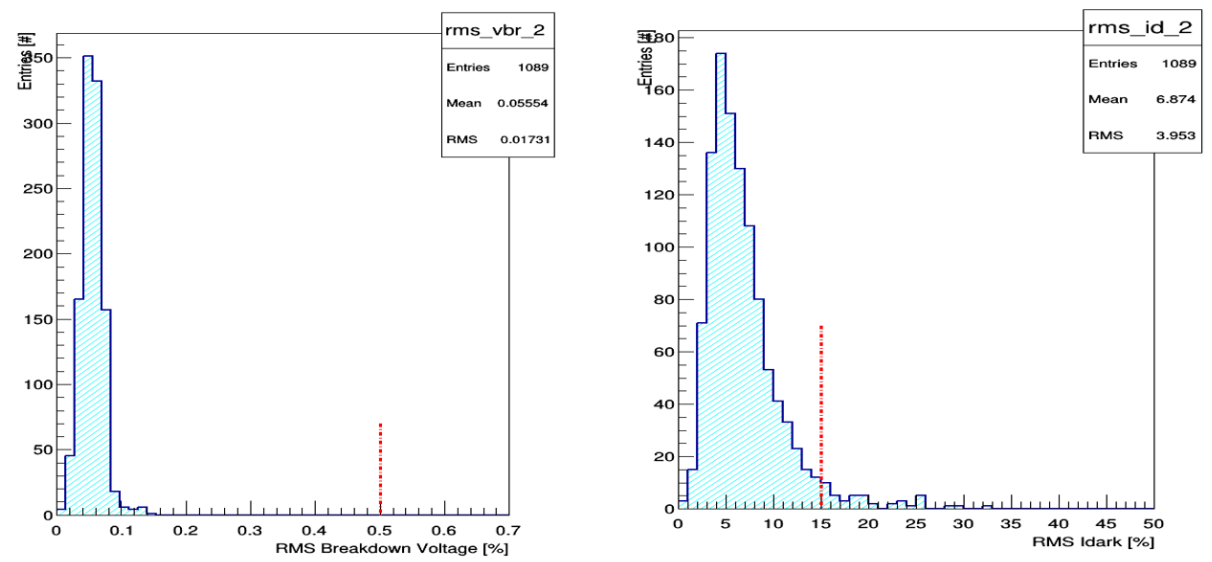

Figure 3: Left - Distribution of the RMS of $\mathrm{V}_{b r}$ within each sensor. Right - Distribution of the RMS of the dark current within each sensor. About the $3 \%$ of the sensors resulted out of the technical specifications.

\section{Conclusions}

The production of the Mu2e calorimeter custom SiPMs is ongoing, proceeding in parallel with the QA. So far, a quarter of the photosensors has been characterized and only the $3 \%$ resulted out of the technical specifications. The overall calorimeter schedule sees the start of the first calorimeter disk assembly in 2019 and completion of its construction in 2020. The qualification of all the photosensors will end in middle 2019.

\section{Acknowledgments}

This work was supported by the EU Horizon 2020 Research and Innovation Programme under the Marie Sklodowska-Curie Grant Agreement No. 690835 and No. 734303.

\section{References}

[1] N. Atanov, et al., The calorimeter of the Mu2e experiment at Fermilab, JINST 12 (01) (2017) C01061. arXiv:1701.07975, doi:10.1088/1748-0221/12/01/C01061.

[2] I. Sarra, et al., The Mu2e Calorimeter Photosensors, Springer Proc. Phys. 213 (2018) 275-279. doi:10.1007/978-981-13-1316-552.

[3] M. Cordelli, et al., Pre-production and quality assurance of the mu2e calorimeter silicon photomultipliers, NIM Adoi:https://doi.org/10.1016/j.nima.2017.12.039.

[4] L. Bartoszek, et al., Mu2e technical design report (2014). arXiv:1501.05241.

[5] N. Atanov, et al., The Mu2e Calorimeter Final Technical Design ReportarXiv:1802.06341.

[6] N. Dinu, et al., Breakdown voltage and triggering probability of sipm from iv curves at different temperatures, NIM A 845 (2017) 64-68. 